Europhysics Letters

PREPRINT

\title{
Intermittency, aging and extremal fluctuations
}

\author{
Paolo Sibani ${ }^{1,2}\left(^{*}\right)$ and HenRIK Jeldtoft Jensen ${ }^{2}$ \\ 1 Theoretical Physics, 1 Keble Rd, Oxford OX1 3NP, UK \\ 2 Dept. of Mathematics, Imperial College London, South Kensington Campus, London \\ $S W r$ 2AZ, UK
}

PACS. 65.60. +a - Thermal properties of amorphous solids and glasses.

PACS. 05.40.-a - Fluctuation phenomena, random processes, noise, and Brownian motion. PACS. 75.10.Nr -Spin-glass and other random models.

\begin{abstract}
Aging in spin glasses is analyzed via the Probability Density Function (PDF) of the heat transfer over a short time $\delta t$ between system and heat bath. The PDF contains a Gaussian part, describing reversible fluctuations, and an exponential 'intermittent' tail. We find that the relative weight of these two parts depends, for fixed $\delta t$ on the ratio of the total sampling time $t$ to the age $t_{w}$. Fixing this ratio, the intensity of the intermittent events is proportional to $\delta t / t_{w}$ and independent of the temperature. The Gaussian part has a variance with the same temperature dependence as the variance of the equilibrium energy in a system with an exponential density of states. All these observations are explained assuming that for any $t_{w}$, intermittent events are triggered by local energy fluctuations exceeding those previously occurred.
\end{abstract}

Introduction. - Aging glassy systems undergo reversible equilibrium-like fluctuations alongside with irreversible configuration changes. As shown experimentally in [1-3] for a number of different cases, these two aspects can be disentangled by a statistical analysis of a mesoscopic noisy signal: within the probability density function (PDF) of the signal, the fluctuations give rise to a Gaussian peak, and the irreversible changes to an asymmetric 'intermittent' tail. This probe of aging dynamics considerably differs from approaches utilizing the fluctuation-dissipation theorem, and raises new theoretical issues regarding e.g. the statistical characterization of the intermittent events and their relationship to the thermal fluctuations. We consider the statistics of the heat-flow over a small interval $\delta t$. As was the case in [1-3], two sub-processes are discernible in the PDF of the data: (i) intermittent events, referred to as quakes, which appear in the left tail of the PDF. These irreversibly release the excess energy trapped by the initial quench. (ii) Gaussian fluctuations which occur between the jumps of type (i) and correspond to reversible energy fluctuations within thermalized attractors. Type (i) events are rare compared to type (ii), but are nevertheless orders of magnitude more frequent than Gaussian fluctuations of similar size. Importantly, the rate of intermittent events decreases with the reciprocal of the age $t_{w}$ and is independent of the temperature $T$. Conversely, the Gaussian fluctuations are $t_{w}$ independent, but strongly $T$ dependent.

$\left({ }^{*}\right)^{1}$ Permanent address: Fysisk Institut, SDU, Odense, DK

(c) EDP Sciences 


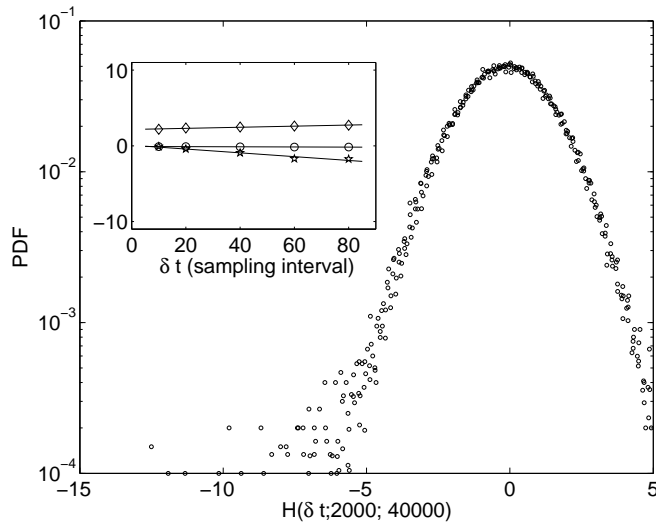

Fig. 1

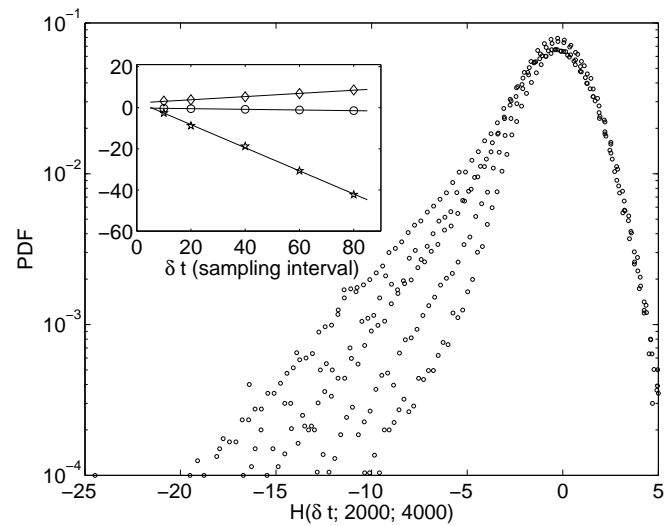

Fig. 2

Fig. 1 - The PDF's (dots) of the heat transfer $H\left(\delta t, t, t_{w}\right)$, sampled in the observation window $\left[t_{w}, t_{w}+t\right]=[40000,42000]$ for $\delta t=10,20,40,60$ and 80 . The temperature is $T=0.3$. Insert: the mean (circles), variance (diamonds) and skewness (stars), are plotted versus the time difference $\delta t$.

Fig. 2 - Data collected as in Fig. 1 but with the observation window $\left[t_{w}, t_{w}+t\right]=[2000,4000]$. A strong asymmetric tail appears, producing a large negative skewness when the observation time $t$ and the age $t_{w}$ are of comparable magnitude.

In this Letter we identify the intermittent events with the large and irreversible configuration changes of glassy systems already described in ref. [4], where they were similarly called quakes. We hence borrow the idea that their occurrence is due to energy fluctuations larger than any hitherto occurred, i.e. fluctuation records and show that record-induced dynamics $[4,5]$ predicts the key dynamical features of the heat transfer PDF. The predictions are first spelled out and tested against simulation data for a prototype glassy system, a three dimensional Ising spin glass with short-ranged couplings. We then argue in greater detail for the theoretical link between record-sized energy fluctuations, density of states within metastable attractors and intermittency, specifically addressing EA spin glasses and other models with short ranged interactions. We conclude with a summary and a brief discussion.

Measurable consequences of record-induced dynamics. - Non-equilibrium aging is described in Ref. [4] in an cartoon-like fashion as a series of irreversible 'quakes' between dynamically inequivalent metastable attractors of an energy landscape. The underlining physical assumptions are: Firstly, that the stability of the 'current attractor', as gauged by an 'exit' barrier, increases by a tiny amount with each quake. Hence a positive energy fluctuation larger than all previous ones, i.e. a record, triggers the irreversible change of attractor. Secondly, that de-facto irreversibility of the quakes arises due to the large energy loss. I.e., crossing extremal barriers triggers irreversible configuration changes, while smaller barriers are crossed reversibly. Thirdly, saddles are visited rarely and in a statistically independent fashion. With these assumptions, the quakes occurring in the interval $\left(t_{w}, t_{w}+t\right]$ are well described by a Poisson process with logarithmic time arguments $[4,8]$, and, by our identification, so are the intermittent events.

The average number of intermittent events is thus

$$
\left\langle n_{I}\left(t_{w}, t_{w}+t\right)\right\rangle=\alpha \log \left(1+t / t_{w}\right), \quad \alpha>0,
$$

where the parameter $\alpha$ slowly increases with the system size but is independent of $T$ [8]. 
Two predictions which are measurable from the PDF of intermittent data without access to microscopic information, are easily derived from Eq. 1.

Firstly, for $\delta t \ll t_{w}$, a single event occurs in the interval $\delta t$ with probability

$$
p_{\left(t_{w}, t_{w}+\delta t\right]}(1)=\alpha \frac{\delta t}{t_{w}}+\mathcal{O}\left(\delta t / t_{w}\right)^{2},
$$

while multiple events have zero probability to linear order in $\delta t / t_{w}$. Thus, the rate of intermittent events decays as $\alpha / t_{w}$ and is independent of the temperature.

Secondly, the number of intermittent events falling in an arbitrary time interval $t$ grows on average with $\log \left(1+t / t_{w}\right)$. As reversible energy fluctuation occur at a constant rate, their number $n_{G}$ in the same interval grows linearly in $t$. The ratio $n_{I} / n_{G}$ is hence given by

$$
\frac{n_{I}}{n_{G}} \frac{t}{t_{w}} \propto \log \left(1+t / t_{w}\right)
$$

The statistical properties of records in a sequence of random numbers are largely insensitive to the distribution from which these numbers are independently drawn [4,5]. This leads to the predicted $T$ independence of the intermittency rate, which is however a puzzling conclusion when thermally activated barrier climbing arguably produces the attractor changes. In the penultimate section of the Letter we analyze this issue in some detail, define the attractors more concretely for a system with short-ranged interactions, and derive Eq. 6] The main result is that the cancellation of strong $T$ dependences needed to ensure consistency between record-dynamics and thermal barrier climbing occurs precisely if the energy fluctuations are drawn from an exponential distribution. In our case, this distribution coincides with the 'local density of states' (LDOS) characterizing the energies available in local thermal equilibrium. Writing the LDOS as

$$
\mathcal{D}(\epsilon) \propto \exp \left(\epsilon / \epsilon_{0}\right)
$$

produces the heat capacity

$$
c_{v}(T) \propto \epsilon_{0}^{2} /\left(\epsilon_{0}-T\right)^{2},
$$

and leads to a Gaussian variance of the heat transfer data

$$
\sigma_{G}^{2} \propto c_{v}(T) T^{2} \propto\left(\frac{\epsilon_{0} T}{\epsilon_{0}-T}\right)^{2}, \quad T<\epsilon_{0} .
$$

This above equation is our third measurable prediction.

Simulation results. - The heat-flow data stem from simulations of a standard $16^{3}$ Edwards-Anderson spin-glass with nearest neighbor Gaussian couplings [6]. For speed we employ an event driven simulation technique [7,8], whose 'intrinsic' time corresponds, for large systems, to the number of Monte Carlo sweeps. The Boltzmann constant is set to one, and age zero is fixed by the instantaneous deep thermal quench preceding the simulations.

We denote by $E\left(t_{w}\right)$ the total energy at age $t_{w}$, pick $\delta t<t_{w}$ and consider a series of $n$ contiguous time intervals of length $\delta t$. For the $k+1$ 'th interval, $k=0,1 \ldots n-1$, the heat $H$ exchanged is calculated as the difference $E\left(t_{w}+(k+1) \delta t\right)-E\left(t_{w}+k \delta t\right)$. Accordingly, $H$ is negative when heat leaves the system. Sampling $H$ over a total observation time $t=n \delta t$ produces the raw data behind the Probability Density Functions (PDFs) discussed below. Since, as it turns out, the form of the PDFs depends on the three parameters $\delta t, t$ and $t_{w}$, the heat transfer is denoted $H\left(\delta t, t, t_{w}\right)$. Omitting the parametric time dependences, the PDF of $H$ is denoted by $p_{H}(x)$, whereas capital letters e.g. $P_{q}$ are used for cumulative distributions. 


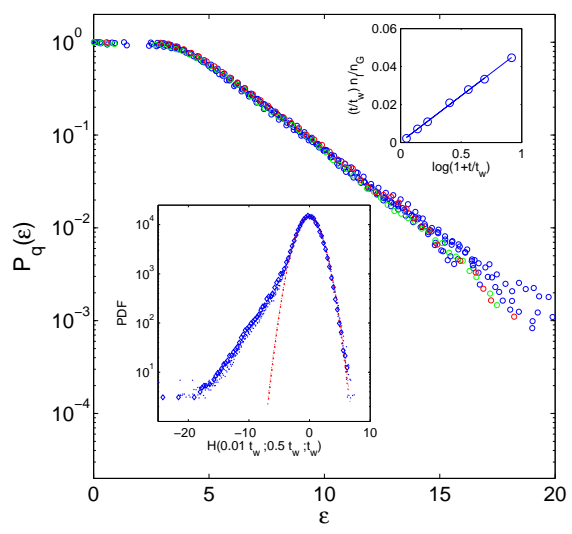

Fig. 3
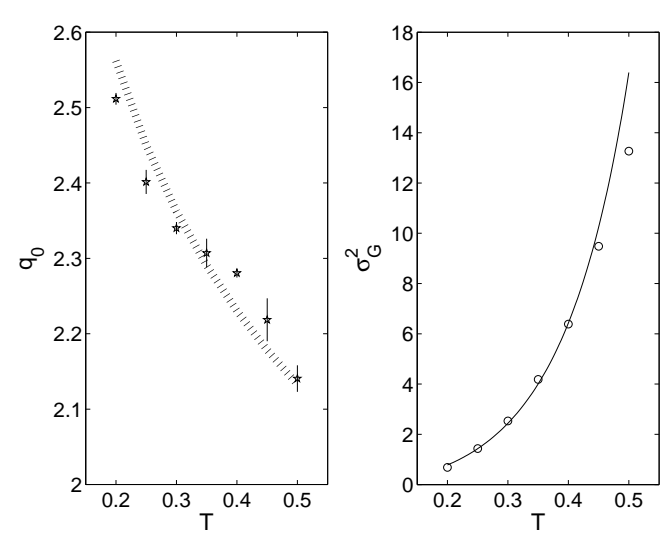

Fig. 4

Fig. 3 - The probability $P_{q}(\epsilon)$ that the amount $q$ of energy intermittently released is larger than $\epsilon$, is plotted versus $\epsilon$ for ages $t_{w}=1000,2000,4000, \ldots 64000$. Lower insert: (unnormalized) PDF of the heat transfer $H\left(0.01 t_{w}, t_{w} / 2, t_{w}\right)$. The full lines-indistinguishable on the scale of the plotare fits to Gaussians with zero average. Upper insert: the relative weight $n_{I} / n_{G}$ (see text) of the intermittent tail is obtained varying $t$ for fixed $\delta t$ from seven values of $H\left(0.005 t_{w}, t, t_{w}\right)$ with $t_{w}=4000$ and $t=200,600,1000,2000,3000,4000$ and 6000 . The straight line is predicted by the theory. The simulation temperature is $T=0.3$.

Fig. 4 - Left frame, symbols: reciprocal slope of $-\log \left(P_{q}(\epsilon)\right)$ for large $\epsilon$, see Fig. 3] as a function of $T$. The dotted band is proportional to $T^{-0.2}$. Right frame, symbols: variance $\sigma_{G}^{2}$ of the Gaussian part of the PDF. Full line: theoretical prediction $\sigma_{G}^{2} \propto T^{2} c_{v}(T)$, with $c_{v}(T)$ given by Eq. 5

To improve the statistics simulations are repeated for 625 or, mainly, 1250 independent runs for each set of physical parameters.

Figures 1 and 2 show data taken for $t=2000, T=0.3$ and five different $\delta t$ 's. In Fig 1 $t_{w}=20 t$ while $t_{w}=t$ in Fig. 2] In agreement with previous observations [1-3,9], the PDF's have a Gaussian part and an exponential tail. In our figures, this tail becomes more pronounced as $t \uparrow t_{w}$, a characteristic shift from equilibrium to non-equilibrium dynamics usually studied through correlations and response functions [10-12].

The asymmetry of the tail shows that large negative $H$ values are associated with irreversible energy losses. These events alone carry the linear $\delta t$ dependence of the mean, variance and skewness shown in the inserts of Figs. 1 and 2 i.e. the Gaussian part of the PDF has always zero average and a variance which depends on $T$ but not on $\delta t$ or $t_{w}$, see e.g. Fig. 2 (NB: the scale differs from Fig 1 ). The linear growth of the variance, also shown in the inserts, continues up to $\delta t \approx t_{w}$ (not shown) and indicates statistical independence of the heat exchange over consecutive time intervals.

For fixed $t / t_{w}$ and increasing $t_{w}$, the rate of intermittent activity decreases as $1 / t_{w}$ in a temperature independent fashion. This $1 / t_{w}$ scaling is shown for $T=0.3 \mathrm{in} \mathrm{Fig.} 3$ where a 64 -fold increase of $t_{w}$ is offset by choosing $\delta t=t_{w} \delta_{0}$ with $\delta_{0}=1 / 100$. The seven $t_{w}$ values considered are $1000 \cdot 2^{n}$, for $n=0,1, \ldots 6$.

The good agreement with the prediction given in Eq. 3is demonstrated in the upper insert of Fig. [3 by plotting $\left(n_{I} / n_{G}\right)\left(t / t_{w}\right)$ versus $\log \left(1+t / t_{w}\right)$. For fixed $\delta t=20$ and $t_{w}=4000$, we consider observation times $t=200,600,1000,2000,3000,4000$ and 10000 (circles). The line 
is a linear fit to the data. The unnormalized PDF's of $H\left(t_{w} / 100, t_{w} / 2, t_{w}\right)$ (dots) and the respective Gaussian fits (overlapping lines) are plotted in the lower insert. The main panel shows the 'tail' distributions $P_{q}(\epsilon)$. For large negative $H$ values these describe the difference $q$ between the empirical PDF value and the Gaussian probability for an energy fluctuation of size $H\left(t_{w} \delta_{0}, t_{w} / 2, t_{w}\right)$. The latter is estimated by fitting a Gaussian of zero average to the 'central part' of the PDF, as delimited by a symmetric cutoff at $\approx \pm 2 \sigma_{G} . P_{q}(\epsilon)$ is later interpreted as the probability that a single quake releases an energy $q$ larger than $\epsilon$. The size distribution of the quakes is nearly exponential, with a decay constant $1 / q_{0}$. The $\delta t / t_{w}$ scaling procedure yielding Fig. 3] was repeated for $T=0.2,0.4$ and 0.5 , with the same result. Additionally, for $T=0.25,0.35,0.45$, six sets of simulations were carried out at fixed $t_{w}=16000$. For each $T$, averaging over the outcomes yields the $q_{0}$ and $\sigma_{G}^{2}$ values plotted in Fig. 4 as circles. The full line in the right panel of Fig. 4 is obtained by Eqs. 6] and 5] using a fitted pre-factor, which amounts to a vertical parallel shift of the theoretical line. The value $\epsilon_{0}=0.86$ used in Eq. 5 is very close to reported values of the critical temperature in 3d Gaussian spin glasses [13].

We note that while the statistical variation of $\sigma_{G}^{2}$ over different simulations set is insignificant, $q_{0}$ is estimated with decreasing accuracy as $T$ and $\sigma_{G}$ increase: the distinction between quakes and fluctuations is moot for $\epsilon$ smaller than $\approx 2 \sigma_{G}$, and the undetermined part of the distribution must be excluded from the fit. With the present numerical effort and accuracy, we can only analyze data below $T=0.6$. The choice of cut-off gives a systematic error, not included in the $\pm \sigma$ error bars. The gray band shows the qualitative agreement with the $T^{-0.2}$ scaling of the energy difference between local minima found in Ref. [8]. This temperature dependence is rather weak compared to the $T$ dependence of $\sigma_{G}^{2}$.

Record fluctuations and intermittency. - In extended systems with short ranged correlations, thermal fluctuations are localized within well separated sub-volumes whose linear size is of the order of the thermal correlation length $[14,15]$. As e.g. suggested in ref [16], each thermalized sub-volume can be treated as a small glassy system, with a 'local' energy landscape. Such landscapes have numerous energy minima. For each minimum $x$, a metastable attractor can be defined as a configuration space neighborhood, e.g. the set of configurations connected to $x$ by paths requiring energy changes below a given threshold [17]. The 'local density of states' is the density of state (DOS) restricted to the configurations belonging to the attractor. LDOS for several glassy systems [17] are indeed well approximated by exponential functions of the energy $\epsilon$ measured from the lowest minimum of the attractor. The divergence of the heat capacity seen in Eq. [5] at $T=\epsilon_{0}$ implies that for $T \geq \epsilon_{0}$ the thermal stability of the attractor vanishes. In practice a rounding off occurs once the weight of configurations outside the attractor becomes important.

The Arrhenius time belonging to the typical extremal barrier crossed at time scale $t_{w}$ is obtained as follows: first we note that the Boltzmann distribution corresponding to an exponential LDOS is itself exponential, and given by $P_{E}(b)=\exp (-b a(T))$, where $a(T)=\left(1 / T-1 / \epsilon_{0}\right)$ is an effective inverse temperature. Secondly, with a constant microscopic attempt rate, the largest fluctuation on a time scale $t_{w}$ is the largest of $\mathcal{O}\left(t_{w}\right)$ values drawn independently from $P_{E}$. Applying a well known mathematical result [18], $b_{\max }\left(T, t_{w}\right) \propto \ln \left(t_{w}\right) / a(T)$. Hence, $a(T)$ cancels, leading to the $T$ independent Arrhenius time $\tau_{A}=\exp \left(a(T) b_{\max }\right)=t_{w}$. A small negative temperature shift imposed at $t_{w}$ generates a mismatch between the extremal barrier previously established and the thermal fluctuations available to cross it. The Arrhenius time and the intermittency rate acquire then a peculiar temperature dependence which has recently been verified in simulations [19].

Clearly, the sum of many independent energy fluctuations is a Gaussian quantity $\left({ }^{1}\right)$, and so

$\left({ }^{1}\right)$ Using a very small $\delta t$ the assumption of independence breaks down, and the Gaussian is replaced by a 
EUROPHYSICS LETTERS

is the central part of the heat transfer PDF, which arises from differences between equilibrium energy fluctuations. The Gaussian variance $\sigma_{G}^{2}$ is therefore twice the equilibrium variance of the energy. The latter follows from Eq. 4 and textbook arguments of equilibrium statistical mechanics.

Finally, figs 13 show how the record dynamics scenario accounts for the dependence of the heat transfer PDF, on $\delta t, t$ and $t_{w}$. Writing the latter as a sum of a Gaussian and an intermittent part, $p_{H} \propto p_{G}+p_{I}$, the tail has the form

$$
p_{I}(x) \propto \log \left(1+t / t_{w}\right) \frac{\delta t}{t_{w}} p_{q}(-x) .
$$

The factor $\frac{\delta t}{t_{w}}$ combines the linear $\delta t$ dependence of the first three moments of $H$ seen in Figs 1 and 2 with the $\delta t / t_{w}$ scaling of the intermittency PDF data shown in Fig. [3] for fixed $t$. The interpretation of $p_{q}(-x)$ as the PDF of the heat transferred in a single quake hinges on $\delta t / t_{w}$ being proportional to the probability of a single intermittent event, see Eq 2

An exponential $p_{q}$ could arise quite simply: the sequence of mainly downhill moves constituting the quake releases an exponentially distributed energy if each move can be the last with equal probability. Lacking a prediction for the weak $T$ dependence of the scale parameter $q_{0}$, we just note its qualitative agreement with the average energy difference between deep minima of the spin-glass landscape [8].

Discussion. - The correspondence between intermittent events and attractor changes was previously investigated in ref. [9], using thermal quenches into inherent structures. We similarly link intermittency to quakes, and derive and verify novel analytical predictions for the heat transfer PDF, based on the assumption that record-sized positive energy fluctuations within the attractors induce the quakes. Record-induced dynamics has generic implications e.g.: the $\delta t / t_{w}$ scaling form of the PDF exemplifies the decelerating dynamics found besides spin-glasses $[11,12,15,20]$ in glasses [21], driven dissipative systems [22, 23], and evolution models $[24,25]$. Nearly exponential densities of states are widespread [26,27], and were used already two decades ago [28] in mesoscopic models of glasses. We thus expect our analysis to broadly apply to glassy systems whenever reversible fluctuations and irreversible quakes are clearly separated, i.e. at sufficiently low temperature. With this limitation, it should also be extensible to other types of measurements, e.g. the fluctuations of two-point correlation functions [1]. Generalizations are needed to account for small but systematic deviations from $t / t_{w}$ scaling, i.e. sub-aging, whose origin is currently debated [29].

Extremal (Gumbel) distributions are ubiquitous, e.g. Chamon et al. [30] finds them to describe local correlators in aging systems, including systems without quenched disorder, and without local energy minima. Since the heat absorbed by an extremal equilibrium fluctuation is masked by the much larger amount of heat released in the following quake, the distribution of extremal energy fluctuations is not directly accessible through intermittent heat-transfer data. The relation with effective temperature descriptions of fluctuation-dissipation data [31, 32 ] warrants a separate discussion. Here we only note that these descriptions concern the asymptotic limit of the fluctuation-dissipation ratio for large times. This regime differs from the one presently considered and is not always reached experimentally $[2,12]$

Acknowledgments-. P.S. thanks J. Dall for his interest, and Pasquale Calabrese, Sergio Ciliberto, Alan Parker, David Sherrington and Veronique Trappe for useful discussions. Financial support from the Danish SNF and from EPSRC is gratefully acknowledged.

back-to back double exponential. 
Paolo Sibani and Henrik Jeldtoft Jensen: Intermittency And AGing

\section{REFERENCES}

[1] Luca Cipelletti, H. Bissig, V. Trappe, P. Ballesta and S. Mazoyer. J. Phys.:Condens. Matter, 15:S257-S262, 2003.

[2] L. Buisson, L. Bellon and S. Ciliberto. J. Phys. Cond. Mat., 15:S1163, 2003.

[3] L. Buisson, S. Ciliberto and A. Garciamartin. Europhys. Lett., 63:603, 2003.

[4] Paolo Sibani and Jesper Dall. Europhys. Lett., 64:8-14, 2003.

[5] P. Sibani and Peter B. Littlewood. Phys. Rev. Lett., 71:1482-1485, 1993.

[6] S. F. Edwards and P. W. Anderson. J. Phys. F, 5:965-974, 1975.

[7] Jesper Dall and Paolo Sibani. Comp. Phys. Comm., 141:260-267, 2001.

[8] Jesper Dall and Paolo Sibani. Eur. Phys. J. B, 36:233-243, 2003.

[9] A. Crisanti and F. Ritort. Europhys. Lett., 66:253-259, 2004.

[10] M. Alba, J. Hammann, M. Ocio, and Ph. Refregier. J. of Appl. Phys., 61:3683-3688, 1987.

[11] J-O. Andersson, J. Mattsson and P. Svedlindh. Phys. Rev. B, 46:8297-8304, 1992.

[12] D. Hérisson and M. Ocio. Phys. Rev. Lett., 88:257202, 2002.

[13] A. P. Young, editor. Spin glasses and random fields. World Scientific, Singapore, New Jersey, London, Hong Kong, 1998.

[14] J. Kisker, L. Santen, M. Schreckenberg, and H. Rieger. Phys. Rev. B, 53:6418-6428, 1996.

[15] Ludovic Berthier and Jean-Philippe Bouchaud. Phys. Rev. B, 66:054404, 2002.

[16] J-M. Drouffe E.M. Bertin, J-P. Bouchaud and C. Godrèche. J. Phys. A, 36:10701-10719, 2003.

[17] P. Sibani, C. Schön, P. Salamon and J.-O. Andersson. Europhys. Lett., 22:479-485, 1993.

[18] M. R. Leadbetter, Georg Lindgren, and Holger Rotzen. Extremes and related properties of random sequences and processes. Springer-Verlag, New York Heidelberg Berlin, 1983.

[19] Paolo Sibani and Henrik Jeldtoft Jensen. JSTAT, page P10013, 2004.

[20] F. Ricci-Tersenghi M. Picco and F. Ritort. Eur.Phys.J. B, 21:211-217, 2001.

[21] W. Kob and J.L. Barrat. European Physical Journal B, 13:319-333, 2000.

[22] P. Sibani and C. M. Andersen. Phys. Rev. E, 64:021103, 2001.

[23] Mario Nicodemi and Henrik Jeldtoft Jensen. J. Phys A, 34:8425, 2001.

[24] P. Sibani and A. Pedersen. Europhys. Lett., 48:346-352, 1999.

[25] Paul Anderson, Henrik Jeldtoft Jensen, L.P. Oliveira and Paolo Sibani. Complexity, 10:49-56, 2004.

[26] P. Sibani and J. C. Schön. In J. Fagerholm, editor, Applied Parallel Computing, Lecture Series in Computer Science, Springer Verlag, 2002.

[27] Tushar S. Jain and Juan J. dePablo. J. Chem. Phys., 116:7238, 2002.

[28] S. Grossmann, F. Wegner, and K. H. Hoffmann. J. Physique Letters, 46:575-583, 1985.

[29] G. F. Rodriguez, G. G. Kenning, and R. Orbach. Phys. Rev. Lett., 91:037203, 2002.

[30] C. Chamon, P. Charbonneau, L. F. Cugliandolo, D. R. Reichman and M. Sellitto. J. Chem. Phys., 121:10120, 2004.

[31] Leticia F. Cugliandolo and Jorge Kurchan and Luca Peliti. Phys. Rev. E, 55:3898-3914, 1997.

[32] P. Calabrese and A. Gambassi. cond-mat041035\%, 2004. 